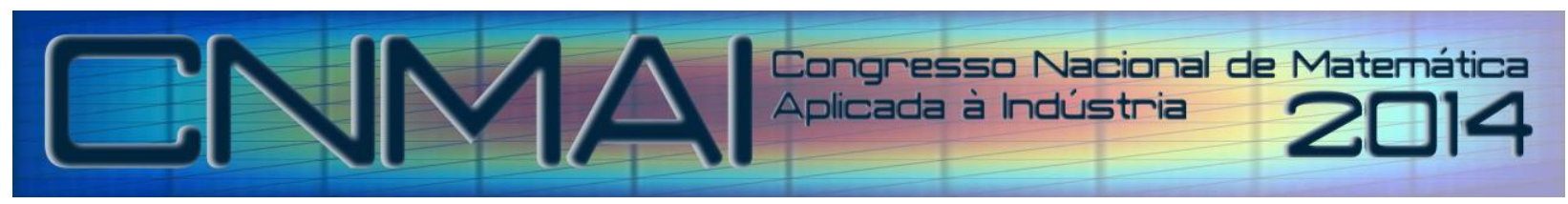

18 a 21 de novembro de 2014, Caldas Novas - Goiás

\title{
ESTUDO DO COMPORTAMENTO DINÂMICO DA EQUAÇÃO DO BALANÇO DE UM SISTEMA DE ENERGIA
}

\author{
Felipe Alves Rubio, felipearubio@gmail.com \\ Fábio Roberto Chavarette, fabioch@mat.feis.unesp.br
}

UNESP - Univ. Estadual Paulista, Faculdade de Engenharia de Ilha Solteira (FEIS) - Departamento de Matemática, Avenida Brasil, 56, Centro, 15385-000 Ilha Solteira, São Paulo.

\begin{abstract}
Resumo: Um dos objetivos da Matemática Aplicada é a busca de soluções para os problemas da sociedade atual. De fato, um produto que incorpore as tecnologias mais avançadas requer que haja soluções inovadoras em todos os segmentos envolvidos no seu desenvolvimento, como por exemplo, no projeto, no uso de materiais inovadores, na produção e nas logísticas de transporte. Em cada uma dessas áreas, os profissionais podem utilizar a modelagem como ferramenta de análise. Os sistemas dinâmicos podem ser estudados em muitas áreas do conhecimento como, por exemplo, no estudo de sistemas biológicos, na eletricidade, na economia, na mecânica e muito outros. Dentro da teoria de sistemas dinâmicos, o estudo da teoria não linear vem ganhando muito destaque, porque a fundamentação teórica existente, juntamente com os modelos já desenvolvidos, pode explicar o comportamento de grande número de exemplos. $O$ sistema que será abordado neste trabalho é a equação do balanço de um sistema de energia, sendo este o conjunto de equipamentos que operam de maneira coordenada de forma a gerar, transmitir e fornecer energia elétrica. $O$ balanço de energia é uma exposição sistemática dos fluxos e transformações de energia em um sistema. A base teórica para um balanço energético é a primeira lei da termodinâmica segundo a qual a energia não pode ser criada ou destruída, apenas modificada em forma. As fontes de energia ou ondas de energia são, portanto, as entradas e saídas do sistema em observação. $O$ objetivo deste trabalho é estudar a estabilidade do sistema não linear e os seus pontos de equilíbrio através do amortecimento do sistema, verificado assim as condições de estabilidade e instabilidade da equação do balanço de um sistema de energia, pensando em obter um melhor desempenho do sistema.
\end{abstract}

Palavras-chave: Sistema de energia, estabilidade, caos

\section{INTRODUÇÃO}

A maioria das atividades humanas envolve vibração de uma forma ou de outra, por exemplo, escutamos devido à vibração dos tímpanos, respiramos devido à vibração dos pulmões e andamos devido ao movimento oscilatório das pernas. Os primeiros estudiosos da área de vibração concentraram seus esforços no entendimento em fenômenos naturais e no desenvolvimento de teorias matemáticas para descrever sistemas físicos. Mais recentemente, muitas investigações foram motivadas pelas aplicações de vibração na área da engenharia, como projeto de máquinas, fundações, estruturas, motores, turbinas e sistemas de controle. A maioria dos motores de acionamento tem problemas de vibração em razão do desbalanceamento inerente aos motores. O desequilíbrio pode dever-se a falha de projeto ou manutenção ruim (Rao, 2008).

Conseguir antecipar um desequilíbrio, ou de modo geral, poder prever um comportamento futuro parece ser extremamente importante, pois minimizar os gastos com reelaborações de projetos devido a falhas encontradas e maximizar o lucro eliminando gastos desnecessários é alvo das grandes indústrias na sociedade atual.

A determinação dos parâmetros de operação ótimos para um processo requer conhecimento profundo sobre o comportamento do sistema. Os balanços de massa e energia são essenciais para o projeto de equipamentos e devem ser satisfeitos, na íntegra, para que um processo seja operado da forma mais econômica possível (Mazzuco, 2013). Desta forma, este trabalho tem o interesse em analisar a equação do balanço de um sistema de energia, verificando em qual situação o sistema apresenta estabilidade ou instabilidade, de acordo com um parâmetro pré-determinado, para poder antecipar um possível colapso ou prever um bom desempenho futuro do sistema.

\section{FORMULAÇÃO DO PROBLEMA MATEMÁTICO}

Na dinâmica do sistema de energia, a atenção está focada, por vezes, em uma única máquina, por conveniência e simplicidade, o resto do sistema é representado por uma barra inifinita onde tensão e frequências são assumidas como constante. 
O estudo do comportamento dinâmico da equação do balanço de um sistema de barramento quase infinito, objeto deste trabalho, é uma simplificação de um sistema de distribuição de energia elétrica, sendo este o conjunto de equipamentos que operam de maneira coordenada de forma a gerar, transmitir e fornecer energia elétrica.

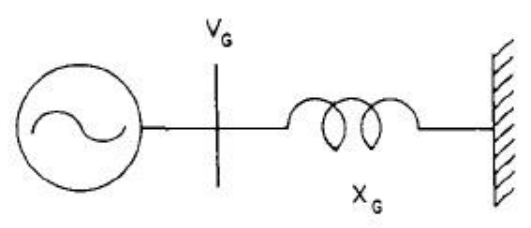

Figura 1. Sistema de Barramento Quase Infinito de uma Maquina Simples (Nayfeh, Balachandran, 2008).

Uma introdução ao problema matemático que se propõe neste trabalho considera-se o sistema de segunda ordem ilustrado na Figura 1 chamado de equação do balanço de um sistema de energia como o seguinte (Nayfeh, Balachandran, 2008):

$$
\frac{2 H}{\omega_{R}} \ddot{\theta}+D \dot{\theta}=P_{m}-\frac{V_{G} V_{B}}{X_{G}} \sin \left(\theta-\theta_{B}\right) .
$$

$\mathrm{Na}$ Eq. (1), $\theta$ representa a posição do rotor do gerador, $\omega_{R}$ representa a velocidade angular, $H$ representa a constante de inércia do rotor, $D$ representa o amortecimento, $P_{m}$ representa a potencia mecânica de entrada, $V_{G}$ é a tensão da máquina, $V_{B}$ é a tensão da barra, $X_{G}$ é a reatância transitória da máquina, $\theta_{B}$ é a fase do barramento, o termo sinusoidal na equação representa à saída de potência elétrica do aparelho e o segundo termo no lado direito da equação representa a energia elétrica gerada (Nayfeh et al, 1990).

Definindo $x_{1}=\theta$ e $x_{2}=\dot{\theta}$, reescrevemos Eq. (1) como o seguinte sistema de equações de primeira ordem:

$$
\left[\begin{array}{l}
\dot{x}_{1} \\
\dot{x}_{2}
\end{array}\right]=\left[\begin{array}{cc}
0 & 1 \\
0 & -D_{c_{1}}
\end{array}\right]\left[\begin{array}{l}
x_{1} \\
x_{2}
\end{array}\right]+\left[\begin{array}{c}
0 \\
P_{m} c_{1}-c_{3} \sin \left(x_{1}-\theta_{B}\right)
\end{array}\right],
$$

sendo $c_{1}=\frac{\omega_{R}}{2 H}$ e $c_{3}=\frac{V_{G} V_{B}}{X_{G}} c_{1}$.

O balanço de energia é uma exposição sistemática dos fluxos e transformações de energia em um sistema. A base teórica para um balanço energético é a primeira lei da Termodinâmica, que é essencialmente um estabelecimento matemático do princípio da conservação da energia aplicada aos sistemas. Esta lei afirma que a variação do conteúdo energético de um sistema fechado é a diferença entre o calor fornecido a este e o trabalho realizado pelo mesmo (Mazzuco, 2013). As fontes de energia ou ondas de energia são, portanto, as entradas e saídas do sistema em observação.

\section{SIMULAÇÃO NUMÉRICA}

O diagrama de estabilidade constitui uma forma bastante sugestiva de representarmos graficamente os pontos de equilíbrio, em função de dois parâmetros convenientes (no nosso caso, $D$ (amortecimento) e $\theta_{B}$ (posição do rotor do gerador)), em que o sistema encontra-se estável ou não.

Para realizar as simulações numéricas do diagrama da estabilidade da Eq. (2) foi implementado um programa no código Matlab®6.5, sendo calculada a matriz de equilíbrio do sistema da Eq. (2), ou seja, a Matriz Jacobiana e calculado os autovalores da matriz considerando-se os valores numéricos para os parâmetros: $H=2.37, V_{B}=1, V_{G}=1.27$, $P_{m}=1, X_{G}=0.645, \omega_{R}=120 \pi, D$ é variado de -1 a 3 e $\theta_{B}$ é variado de 0 a 0,1 . Os autovalores indicam se o comportamento do sistema encontra-se estável ou não de acordo com sua classificação (Monteiro, 2011). 


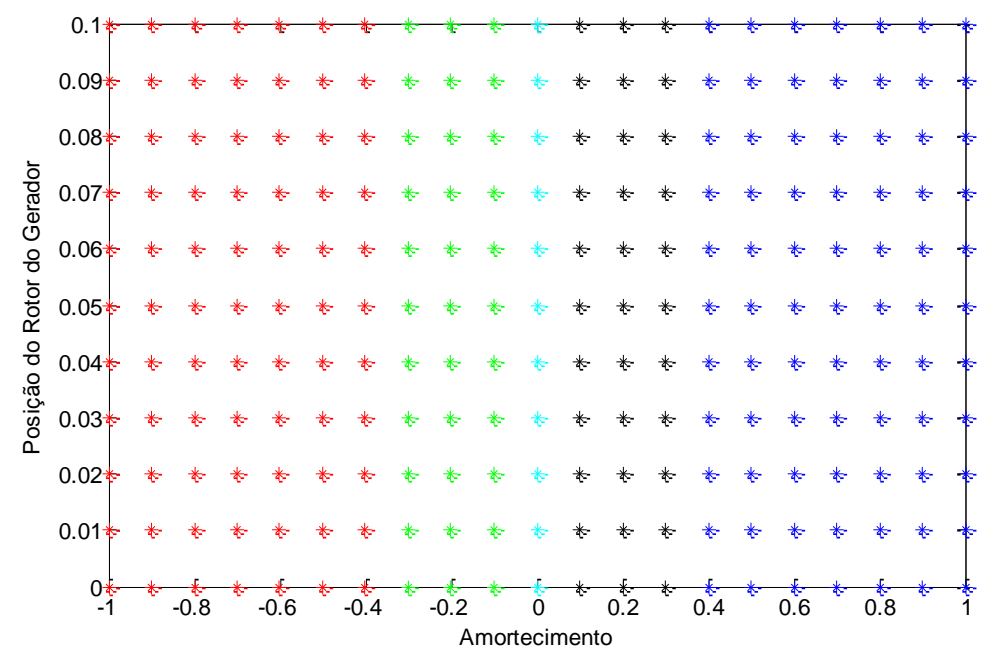

Figura 2. Diagrama de estabilidade da Equação do Balanço do Sistema de Energia. Pontos de Equilíbrio do Sistema. (*) nó hiperbólico instável; (*) focos hiperbólicos instáveis; (*) centro elípticos estáveis; $(*)$ focos hiperbólicos estáveis e (*) nó hiperbólico estável;

A Figura 2 ilustra o diagrama de estabilidade do sistema dinâmico da Eq. (2) sendo os pontos de equilíbrio com sua respectiva classificação e estabilidade. Os pontos em vermelho e azul representam nós hiperbólicos, instáveis e estáveis respectivamente. Os pontos em verde e preto são focos hiperbólicos, sendo os pontos em verde instáveis e os pontos em preto estáveis. Os pontos em ciano são centros elípticos estáveis.

A seguir demonstraremos três comportamentos dinâmicos distintos, sendo o amortecimento $(D)$ nulo, positivo e negativo.

\subsection{Centro Elíptico Estável.}

Para demonstrar o comportamento estável do tipo centro elíptico na Figura 3, adota-se o parâmetro $D$ como sendo um valor nulo e utilizando-se o integrador ODE45, considerando-se os valores numéricos para os parâmetros: $H=2,37$, $V_{B}=1, V_{G}=1,27, P_{m}=1, X_{G}=0,645, \omega_{R}=120 \pi$ e $\theta_{B}=0$.

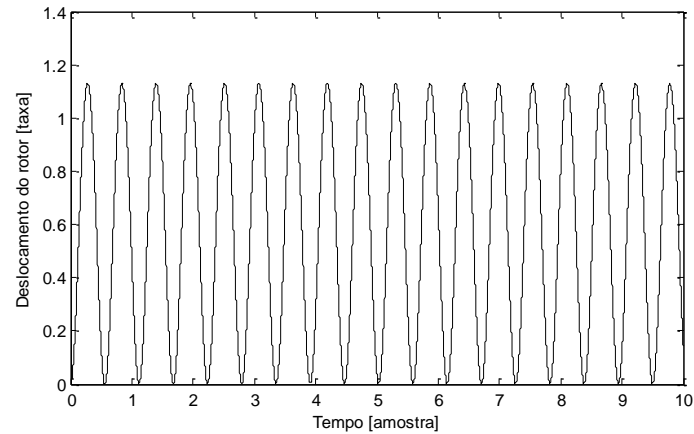

(a) (b)

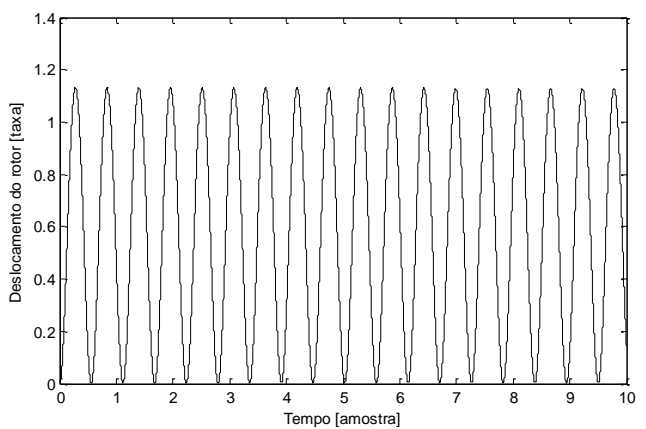

(c)

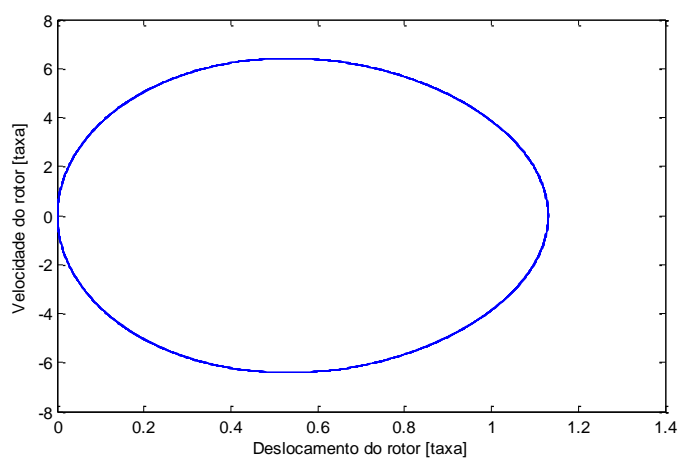

Figura 3. Comportamento Estável. (a) Histórico no Tempo para o Deslocamento do Rotor, (b) Histórico no Tempo para a Velocidade do Rotor e (c) Retrato de Fase da Equação do Balanço do Sistema de Energia. 
O ponto de equilíbrio do sistema é $(0,58272,0,0000)$, sendo que este possui um comportamento instável conforme os autovalores do sistema $\left(\lambda_{1,2}=0,000 \pm 11614 i\right)$. Os autovalores do sistema indicam que o sistema possui um foco hiperbólico instável.

\subsection{Foco Hiperbólico Estável.}

Novamente, para demonstrar o comportamento estável do tipo foco hiperbólico na Figura 4, adota-se o parâmetro $D$ como sendo um valor positivo $(D=0,001)$ e considerando-se os parâmetros como citados anteriormente.

O ponto de equilíbrio do sistema é $(0,58272,0,0000)$, sendo que este possui um comportamento estável conforme os autovalores do sistema $\left(\lambda_{1,2}=-0,0358 \pm 116145 i\right)$. Os autovalores do sistema indicam que o sistema possui um foco hiperbólico estável.

(a)

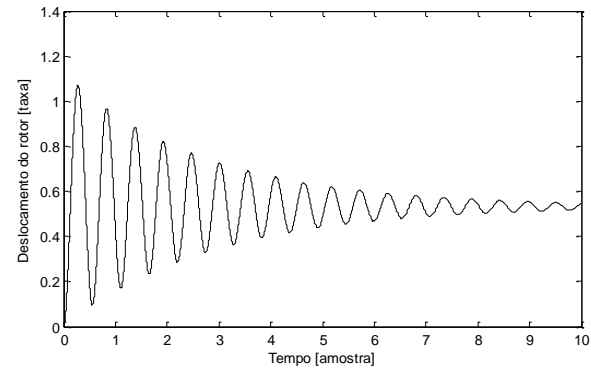

(b)

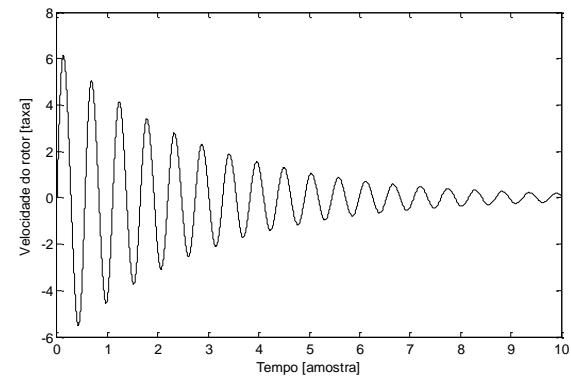

(c)

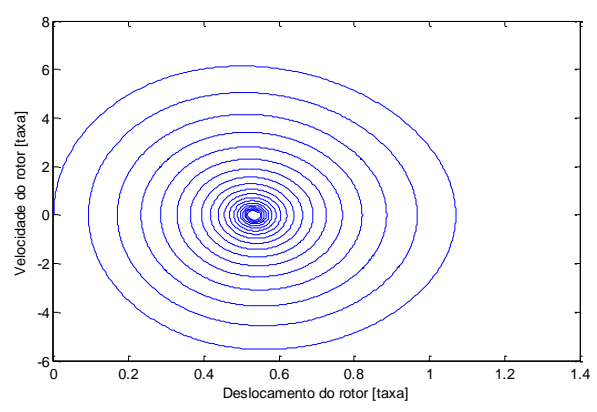

Figura 4. Comportamento Estável. (a) Histórico no Tempo para o Deslocamento do Rotor, (b) Histórico no Tempo para a Velocidade do Rotor e (c) Retrato de Fase da Equação do Balanço do Sistema de Energia.

\subsection{Foco Hiperbólico Instável e Caótico.}

O último comportamento a ser demonstrado, o comportamento instável do tipo foco hiperbólico como mostra a Figura 5, adota-se o parâmetro $D$ como sendo um valor negativo $(D=-0,001)$ e considerando-se os parâmetros como citados anteriormente.

(a)

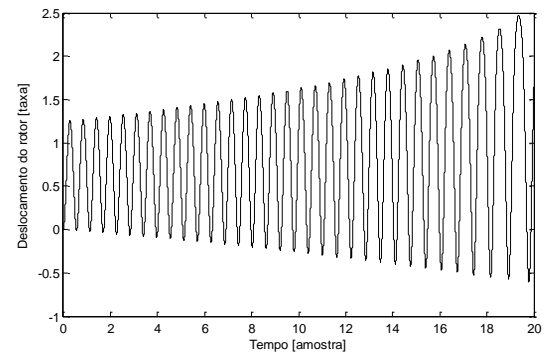

(b)

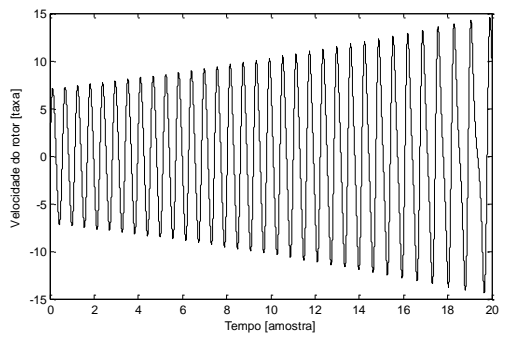

(c)

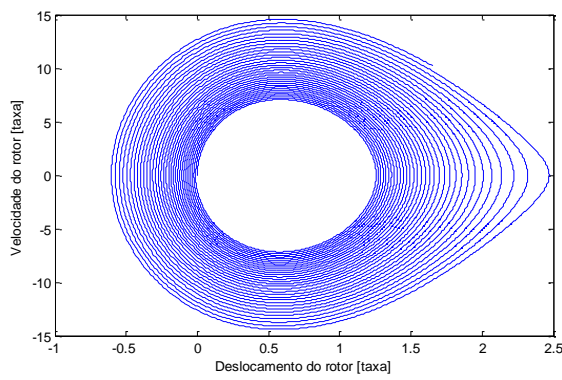

Figura 5. Comportamento Instável. (a) Histórico no Tempo para o Deslocamento do Rotor, (b) Histórico no Tempo para a Velocidade do Rotor e (c) Retrato de Fase da Equação do Balanço do Sistema de Energia. 
O ponto de equilíbrio do sistema é $(0,58272,0,0000)$, sendo que este possui um comportamento instável conforme os autovalores do sistema $\left(\lambda_{1,2}=0,03976 \pm 11614 i\right)$. Os autovalores do sistema indicam que o sistema possui um comportamento instável como mostrado na Figura 2.

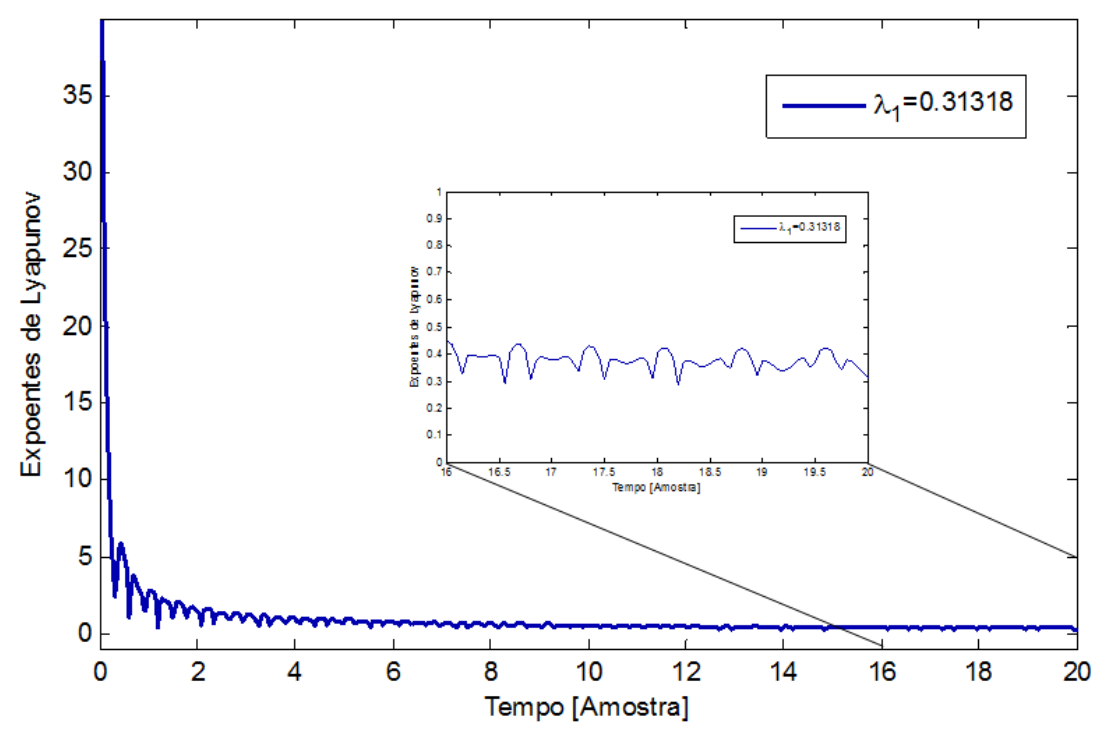

Figura 6. Expoente de Lyapunov da Equação do Balanço do Sistema de Energia.

A Figura 6 mostra a dinâmica do comportamento instável caótico dos expoentes de Lyapunov $\left(\lambda_{1}=0,31318\right)$, (Wolf et $a l, 1985)$, tal modificação no comportamento do sistema se deu a aplicação inclusão de um pequeno valor negativo no amortecimento do sistema.

Este comportamento ilustrado na Figura 6 muitas vezes causa um mau funcionamento em diversos dispositivos podendo levar a prejuízos econômicos. No caso de um sistema de energia, o mesmo poderá entrar em colapso na geração de energia e a instabilidade pode causar grandes danos ao sistema.

\section{CONCLUSÃO}

Neste trabalho, a dinâmica da equação do balanço de um sistema de energia proposto (Nayfeh, Balachandran, 2008) é investigada através de simulações numéricas usando o software Matlab $6.5^{\circledR}$. O modelo é mostrado na seção 2 e a estabilidade é demonstrada e analisada na seção 3.

O diagrama de estabilidade ilustrado na Figura 2 demonstra os comportamentos estáveis e instáveis do sistema de energia conforme Eq. (2). Esse diagrama é importante, pois demonstra que o efeito do amortecimento no sistema, mesmo que pequeno, torna o comportamento do sistema estável, sendo este um comportamento necessário para um bom desempenho do mesmo.

As Figuras 3 e 4 mostram o comportamento estável do sistema proposto, estes comportamentos são os comportamentos esperados para um bom desempenho do sistema de energia. Para demonstramos o comportamento instavel do sistema, foi introduzido um pequeno amortecimento negativo sendo o suficiente para este objetivo, como mostra a Figura 5. A Figura 6 ilustra o expoente de Lyapunov para o modelo com a presença de caos, ou seja, a instrodução do valor negativo também causa um colapso no sistema de energia.

O estudo da estabilidade do comportamento dinâmico da equação do balanço de um sistema de energia, objeto deste trabalho, sendo o sistema o conjunto de equipamentos que operam de forma a gerar, transmitir e fornecer energia de maneira coordenada, é de extrema importância para se prever um bom desempenho futuro do sistema.

\section{AGRADECIMENTOS}

Os autores agradecem à FAPESP (Proc. No. 2014/04248-0) e ao CNPq (Proc. No. 301769/2012-5) pelo apoio financeiro desta pesquisa. 


\section{REFERÊNCIAS}

Mazzuco, M. M. 2013. Introdução aos Balanços de Massa e Energia. Notas de Aula. 111 p.

Monteiro, L. H. A. 2011. Sistemas Dinâmicos, São Paulo, Brazil: Ed. Livraria da Física. 670 p.

Nayfeh, A., Balachandran, B., 2008. Applied Nonlinear Dynamics: Analytical, Computational and Experimental Methods. New York: Ed. John Wiley \& Sons, 691p.

Nayfeh, M. A., Hamdan, A. M. A., Nayfeh A. H., 1990. Chaos and Instability in a Power System - Primary Resonant Case, Nonlinear Dynamics, (1), 313-339.

Rao, S. S. 2008. Vibrações Mecânicas. São Paulo, Brazil: Ed. Pearson Prentice Hall.. 424 p.

Wolf, A., Swift, J. B., Swinney, H. L., Vastano, J. A., 1985. Determining Lyapunov Exponents from a Time Series. Physica D, (16), 285-317.

\section{RESPONSABILIDADE AUTORAL}

Os autores são os únicos responsáveis pelo conteúdo deste trabalho. 


\title{
STUDY OF THE DYNAMIC PERFORMANCES OF THE SWING EQUATION OF A POWER SYSTEM
}

\author{
Felipe Alves Rubio, felipearubio@gmail.com \\ Fábio Roberto Chavarette, fabioch@mat.feis.unesp.br
}

UNESP - Univ. Estadual Paulista, Faculdade de Engenharia de Ilha Solteira (FEIS) - Departamento de Matemática, Avenida Brasil, 56, Centro, 15385-000 Ilha Solteira, São Paulo.

\begin{abstract}
One goal of Applied Mathematics is the search for solutions to the contemporary society. Indeed, a product that incorporates the most advanced technologies requires that there innovative solutions in all segments involved in its development, for example, in use innovative materials, in production and logistics transportation. In each of those areas, the professionals can use the modelling as an analysis tool. The dynamic systems can be studied in many areas as, for example, in the study of biological systems, electricity, economics, mechanics and many others. Within the theory of dynamical systems, the study of nonlinear theory has gained much prominence, because the existing theoretical foundation, along with the models already developed, can explain the behavior of large numbers of examples. The system will be addressed is the swing equation of an energy system, and an electric energy system the set of equipment that operate in a coordinated manner in order to generate, transmit and supply electricity. The energy swing is a systematic exposition of the flows and transformations of energy in a system. The theoretical basis for an energy balance is the first law of thermodynamics according to which energy can't be created or destroyed, only changed in form. Energy sources or energy waves are, therefore, the inputs and outputs of the system under observation. The objective of this work is to study the stability of the proposed system and its equilibrium points through the control parameter D, which represents the damping of the system, verified so the conditions of stability and instability of the swing equation of a power system, thinking of getting a better control of the system.
\end{abstract}

Keywords: Power system, stability, chaos 